\title{
Espessura e qualidade musculares medidas a partir de ultrassonografia: influência de diferentes locais de mensuração
}

\section{Muscle thickness and muscle quality measured by ultrasound: influence of different sites of measurement}

Regis Radaelli

Eurico Nestor Wilhelm Neto

Martim Francisco Bottaro Marques

Ronei Silveira Pinto

1 Universidade Federal do Rio Grande do Sul. Escola de Educação Física. Porto Alegre, RS. Brasil.

2 Universidade de Brasília. Faculdade de Educação Física. Brasília, DF. Brasil.

Enviado em 22/06/10 Revisado em 26/10/10 Aprovado em 05/12/10
Resumo - Os objetivos do presente estudo foram: a) observar se há diferença entre os valores de espessura muscular (EM) medidos por ultrassonografia e qualidade muscular (QM) de três diferentes pontos nos músculos flexores de cotovelo e extensores de joelho, b) analisar a correlação das medidas de EM e força isométrica de flexão de cotovelo e extensão de joelho. Sessenta e nove sujeitos homens, fisicamente ativos, participaram do estudo. Os valores de produção de força isométrica de flexão de cotovelo e extensão de joelho foram obtidos nos ângulos de $90^{\circ}$ e $60^{\circ}$, respectivamente. A medida de EM foi realizada em três pontos nos flexores de cotovelo (proximal - BB1, médio - BB2, e distal - BB3) e extensores de joelho (proximal - RI1, medial - RI2, e distal - RI3). As diferenças entre as três medidas de EM e QM foram avaliadas por meio da one-way ANOVA para medidas repetidas, e a correlação entre EM e produção de força foi testada utilizando correlação de Pearson. O nível de significância utilizado foi de $\mathrm{p}<0,05$. A EM e a QM diferiram estatisticamente nos três pontos de medidas, tanto nos flexores do cotovelo quanto nos extensores do joelho $(\mathrm{p}<0,001)$. As correlações entre as medidas de EM dos flexores de cotovelo nos pontos BB1, BB2 e BB3 e a produção de força isométrica foram $r=0,57, r=0,52$ e $r=0,59(p<0,05)$, respectivamente. Já nos extensores de joelho, estas foram de $r=0,47, r=0,51$ e $r=0,53$ ( $p<0,01$ ), nos pontos RI1, RI2, e RI3, respectivamente. Os resultados do presente estudo demonstraram que o ponto de medida exerce influência significativa na EM e QM. Os valores de EM relacionam-se com os de produção de força isométrica dos flexores de cotovelo e extensores de joelho.

Palavras-chave: Ultrasonografia; Espessura muscular; Qualidade muscular; Força muscular.

Abstract - The aims of this study were: a) to investigate differences in muscle thickness (MT) measured by ultrasound and muscle quality (MQ) at three sites in the elbow flexors and knee extensors, and b) to correlate MT with isometric force production of the elbow flexors and knee extensors. Sixty-nine young male subjects participated in this study. The isometric force production values of the elbow flexors and knee extensors were obtained at $90^{\circ}$ and $60^{\circ}$, respectively. MT was assessed at three sites in the elbow flexors (proximal-BB1, middle-BB2, and distal-BB3) and knee extensors (proximal-RI1, middle-RI2, and distal-RI3). Differences between the three measurements of MT and MQ were evaluated by one-way repeated measures ANOVA. Pearson's correlation test was used to assess the correlation between MT and isometric force. The level of significance was set at $p<0.05$. The results showed significant differences $(p<0.001)$ between the three MT and MQ sites in the elbow flexors (BB1, BB2, BB3) and in the knee extensors (RI1, RI2, RI3). The correlation between BB1, BB2 and BB3 and force production was $r=0.57, r=0.52$ and $r=0.59$, respectively $(p<0.05)$. In the knee extensors, the correlation between RI1, RI2 and RI3 and force production was $r=0.47, r=0.51$ and $r=0.53$, respectively $(p<0.01)$. There were significant differences in $M Q$ between elbow flexors $(p<0.001)$ and knee extensors $(p<0.001$ The present results showed that the site of measurement has a significant influence on MT and MQ. Furthermore, MT was positively correlated with isometric force production in the elbow flexors and knee extensors.

Key words: Ultrasonography; Muscle thickness; Muscle quality; Muscle strength. 


\section{INTRODUÇÃO}

A produção de força está relacionada a variáveis neurais e morfológicas ${ }^{1}$. As principais variáveis morfológicas são o percentual dos diferentes tipos de fibras musculares ${ }^{2}$ a área de secção transversa muscular $^{3}$ (AST), o volume muscular ${ }^{4}$, bem como a espessura muscular ${ }^{5,6}$ (EM). A avaliação das variáveis morfológicas tem sido realizada por inúmeros métodos, como por exemplo, a biópsia muscular, a ressonância magnética e a tomografia computadorizada. Entretanto, devido ao seu menor custo em relação à ressonância magnética e à tomografia, além de ser um método de avaliação não invasivo, a ultrassonografia vem sendo apresentada como uma alternativa para a avaliação de algumas variáveis morfológicas envolvidas na produção de força, como o ângulo de penação, a AST, e a EM ${ }^{5,7-9}$.

A utilização da EM por meio da ultrassonografia tem sido frequentemente utilizada em investigações com o intuito de avaliar as adaptações crônicas musculares em resposta a diferentes protocolos de treinamento de força ${ }^{10,11}$, sendo, muitas vezes, também associada à produção de força muscular decorrente do treino5. A qualidade muscular (QM) parece ser também uma medida importante, pois reflete a capacidade funcional muscular. A QM, também referida como tensão específica, refere-se à produção de força por unidade de área muscular ${ }^{12,13}$. Sua utilização pode dar suporte à observação de adaptações morfológicas e neurais em resposta a um programa de treinamento, destreino e envelhecimento, e ainda parece ser uma medida mais interessante do que apenas o valor de produção de força muscular, pois pode representar a capacidade funcional muscular. Geralmente, a unidade muscular usada para o cálculo de QM é a AST ou o volume muscular ${ }^{13,14}$. No entanto, a EM pode também ser utilizada na avaliação da QM, já que sua medida tem uma estreita relação com a $\mathrm{AST}^{15}$. Além disso, a EM é uma das variáveis que, juntamente com o comprimento do membro, exerce mais influência sobre o cálculo do volume muscular ${ }^{16}$.

Os procedimentos metodológicos adotados na mensuração da EM incluem, entre outros, a determinação criteriosa dos pontos anatômicos onde será realizada a medida. Porém, diferentes metodologias vêm sendo utilizadas no que diz respeito aos pontos de mensuração da $\mathrm{EM}^{5,10,11}$. Nesta perspectiva, a uniformidade dos pontos de medida da EM parece ter relevância quer em estudos transversais quer em estudos longitudinais que se utilizam desta variável. Como exemplo disso, têm sido identificadas na literatura adaptações morfológicas diferenciadas ao longo do músculo, em resposta a programas de treinamento, que avaliaram a EM por ultrassonografia ${ }^{10,17,18}$. Portanto, a comparação entre investigações que realizaram a ultrassonografia para avaliar alterações morfológicas musculares, bem como a QM, podem ser comprometidas, uma vez que diferentes pontos de medida da EM podem representar diferentes adaptações morfológicas e valores distintos de QM.

Sendo assim, os objetivos do presente estudo foram: a) observar se há diferença entre os valores de espessura muscular (EM) medidos por ultrassonografia e qualidade muscular (QM) de três diferentes pontos nos músculos flexores de cotovelo e extensores de joelho, b) analisar a correlação das medidas de EM e força isométrica de flexão de cotovelo e extensão de joelho.

\section{PROCEDIMENTOS METODOLÓGICOS}

\section{Sujeitos}

O presente estudo contou com um total de 69 voluntários $(26,9 \pm 4,4$ anos; $67,7 \pm 8,1 \mathrm{Kg} ; 169,1$ $\pm 5,8 \mathrm{~cm})$. Os sujeitos inclusos na amostra foram homens fisicamente ativos que praticavam atividade física, pelo menos, três vezes por semana há, pelo menos, seis meses, e que não tinham restrições para realizar os testes de avaliacão de forca, sendo que, devido a impossibilidades técnicas, apenas 39 tiveram a EM e a produção de força dos flexores de cotovelo avaliada, e 30 tiveram a EM dos extensores de joelho avaliada e realizaram o teste para avaliação de força destes grupos musculares. Todos os sujeitos assinaram um termo de consentimento livre e esclarecido e estavam cientes dos objetivos do estudo, bem como de seus riscos e benefícios. Este estudo foi aprovado pelo comitê de ética da Universidade Federal do Rio Grande do Sul (n 15723).

\section{Contração isométrica voluntária máxima (CIVM)} de flexão de cotovelo e extensão de Joelho

A produção de força dos indivíduos foi avaliada por meio de CIVM (pico de torque, N.m). Para a coleta do valor de pico de torque (PT, em N.m) de flexão de cotovelo e extensão de joelho, foi utilizado um dinamômetro isocinético (Cybex Norm, Ronkonkoma, NY, EUA), o qual foi calibrado de acordo as instruções do fabricante.

Para o teste de flexão de cotovelo, os sujeitos foram posicionados em decúbito dorsal com o ombro abduzido em $45^{\circ}$, o qual também foi estabilizado por um velcro. $O$ centro de rotação do dinamômetro foi alinhado com o epicôndilo lateral do úmero. Para esse 
teste, cada indivíduo realizou três CIVMs no ângulo de $90^{\circ}$ de flexão de cotovelo, durante cinco segundos, com um período de recuperação de cinco minutos. Antes do teste, foi realizada a correção da gravidade. Os sujeitos receberam estímulo verbal e visual durante o teste. Na realização do teste de extensores do joelho, era solicitado aos voluntários que mantivessem seus braços cruzados na altura do tórax ${ }^{27}$.

Para a obtenção dos valores de CIVM de extensão de joelho, os sujeitos sentaram com o quadril flexionado em $85^{\circ}\left(0^{\circ}\right.$ referente ao quadril completamente estendido) e foram estabilizados por um cinto, colocado em volta do peito, e por um velcro, passado em torno da coxa do membro que realizou o teste. Para a execução dos testes, o epicôndilo lateral do fêmur foi alinhado visualmente com o eixo de rotação do dinamômetro e o braço de alavanca do equipamento foi ajustado para cada sujeito. Antes do teste, também foi realizada a correção da gravidade. O protocolo de testes consistiu em três CIVMs, de cinco segundos de duração, no ângulo de $60^{\circ}\left(0^{\circ}\right.$ extensão completa de joelho), com cinco minutos de intervalo entre cada CIVM. Os sujeitos receberam estímulo verbal visual durante o teste. Para análise estatística, foi utilizado somente o maior valor de PT de flexão de cotovelo e extensão de joelho das CIVMs, e somente do lado dominante.

\section{Avaliação da espessura muscular (EM)}

A avaliação da EM (mm) foi feita por meio de imagem obtida com aparelho de ultrassonografia (Philips, VMI, Indústria e Comércio Ltda. Lagoa Santa, MG, Brasil), sendo a imagem obtida em B-modo. Durante a avaliação da EM, os sujeitos permaneceram deitados com o membro avaliado estendido e relaxado. Um transdutor com frequência de amostragem de $7,5 \mathrm{MHz}$ foi posicionado de forma perpendicular sobre os músculos avaliados. Para a aquisição da imagem, foi utilizado um gel à base de água, que promove um contato acústico sem a necessidade de causar pressão sobre a pele. Durante o teste, foi evitado qualquer tipo de pressão do transdutor sobre a pele dos sujeitos. $\mathrm{O}$ tecido adiposo subcutâneo e tecido ósseo foram identificados pela imagem da ultrassonografia, e a distância entre esses dois pontos foi definida como a EM. A avaliação da EM foi efetuada no segmento dominante e todas as medidas foram executadas pelo mesmo avaliador.

O ponto principal de avaliação dos músculos bíceps braquial e braquial foi realizado de acordo com a proposição de $\mathrm{Abe}^{5}$, a $60 \%$ da distância entre o epicôndilo lateral do úmero e o processo acromial do rádio, e foi definido como BB3 (ponto distal). Além desse, mais dois pontos foram utilizados para as correlações: BB2 e BB1, demarcados um centímetro e meio e três centímetros acima de $\mathrm{BB} 3$, respectivamente (figura 1).

O ponto principal utilizado para a medida da EM dos músculos reto da coxa e vasto intermédio (ponto médio) foi definido de acordo com a proposição de Chilibeck ${ }^{19} \mathrm{e}$ foi definido como RI2. Além desse ponto de mensuração, mais dois pontos foram demarcados cinco centímetros acima (RI1) e abaixo (RI3) (Figura 2).

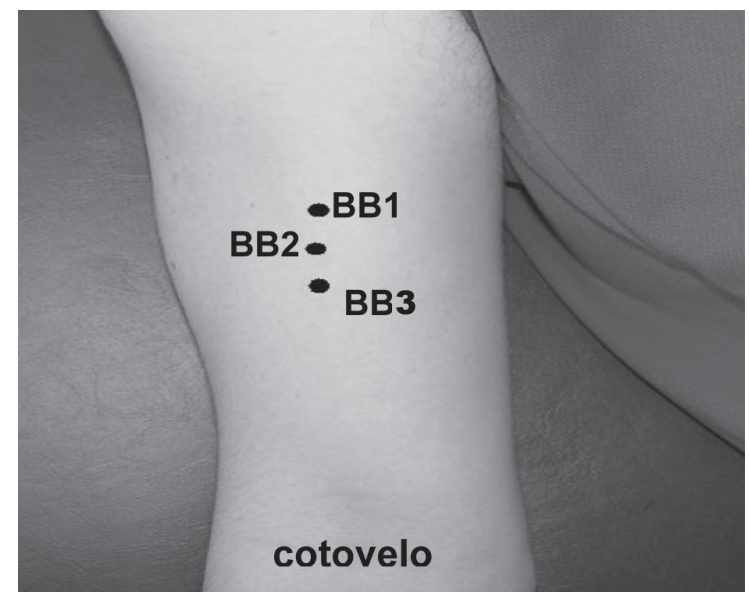

Figura 1. Pontos sobre os flexores de cotovelo.

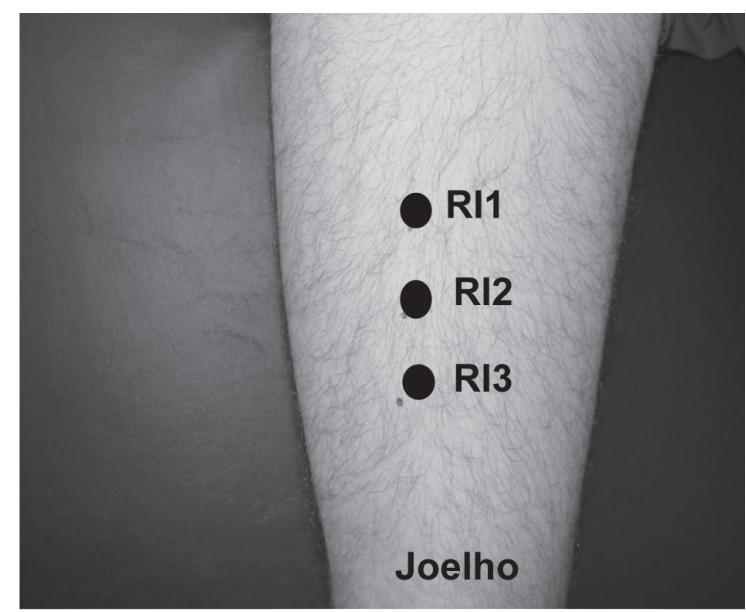

Figura 2. Pontos sobre os extensores do joelho.

A QM foi calculada para cada ponto de avaliação da EM dos flexores de cotovelo e extensores de joelho. Esse valor foi obtido a partir da divisão do valor de produção de força isométrica de flexores de cotovelo pelos valores de EM dos pontos BB1, BB2 e BB3 e pela divisão do valor de produção de força isométrica dos extensores de joelho pelos valores de EM dos pontos RI1, RI2 e RI3.

\section{Análise Estatística}

Os dados obtidos passaram por análise estatística para a verificação de sua normalidade por meio do 
teste de Shapiro-wilk. Após os dados demonstrarem distribuição normal, foi utilizado o Coeficiente de Correlação de Pearson para correlacionar os valores de PT das CIVMs de flexão de cotovelo e extensão de joelho com os com seus respectivos valores de EM. Para a comparação entre os valores de EM obtidos dos diferentes pontos em cada músculo e da QM calculada a partir desses valores, foi utilizada one-way ANOVA de três fatores para medidas repetidas e o teste post hoc Bonferoni para a localização das diferenças. Todos os dados foram analisados no programa SPSS 13.0 e foi adotado o nível de significância de $\mathrm{p}<0,05$.

\section{RESULTADOS}

Os valores médios de pico de torque, espessura muscular e qualidade muscular estão apresentados na tabela 1 (média $\pm \mathrm{DP}$ ).

Os valores da espessura muscular do ponto BB3 foram significativamente maiores que os valores medidos nos pontos BB2 e BB1 ( $<<0,001)$; sendo os valores do ponto $\mathrm{BB} 2$ significativamente maiores que os do BB1 $(p<0,001)$. Em relação aos pontos em que a espessura muscular foi mensurada nos extensores do joelho, os valores do ponto RI1 foram significativamente maiores que os dos pontos RI2 e RI3 ( $p<0,001$ ), e os valores do ponto RI2 foram significativamente maiores que os do ponto RI3 $(\mathrm{p}<0,001)$. Relativamente à qualidade muscular, houve diferença significativa entre os valores desta variável obtidos a partir dos pontos de medição da espessura muscular. A qualidade muscular calculada a partir do ponto BB3 foi significativamente maior $(p<0,001)$ que a qualidade muscular dos pontos BB2 e BB1, sendo que a qualidade muscular do ponto BB2 foi significativamente maior $(p<0,001)$ que a do BB1. Já para os extensores do joelho, os valores da qualidade muscular calculados a partir do ponto RI1 foram significativamente maiores $(\mathrm{p}<0$, 001) que os valores dos pontos RI2 e RI3, sendo os valores do ponto RI2 significativamente maiores $(\mathrm{p}<0,001)$ que os do RI3 (Tabela 1).

As correlações entre os valores de produção de força isométrica dos flexores de cotovelo e extensores de joelho e os valores da espessura muscular destes músculos, realizadas no presente estudo, demonstraram ser estatisticamente significativas $(\mathrm{p}<0,05)$. Assim, todos os pontos foram utilizados para o cálculo da qualidade muscular. As correlações entre os valores de PT na flexão de cotovelo e os valores da espessura muscular nos pontos BB1, BB2 e BB3 foram de $r=0,57, r=0,52$ e $r=0,59$ $(p<0,05)$, respectivamente (Figura $3 A ; B ; C)$.

As correlações entre os valores de PT na extensão de joelho e os valores da espessura muscular nos pontos RI1, RI2 e RI3 foram de $\mathrm{r}=0,47, \mathrm{r}=0,51 \mathrm{e}$ $\mathrm{r}=0,53,(\mathrm{p}<0,01)$, respectivamente (Figura 4A; B; C).

Tabela 1. Valores médios de pico de torque, espessura muscular e qualidade muscular, dos flexores de cotovelo e extensão de joelho.

\begin{tabular}{lccccccc}
\hline & Pico de Torque $(\mathrm{Nm})$ & \multicolumn{3}{c}{ Espessura Muscular $(\mathrm{mm})$} & & \multicolumn{2}{c}{ Qualidade Muscular } \\
\hline & & & & & & & \\
& & BB1 & BB2 & BB3 & BB1 & BB2 & BB3 \\
Flexores de cotovelo & $64,3 \pm 11,5$ & $31,9 \pm 2,9$ & $34,3 \pm 3,3^{*}$ & $36,5 \pm 3,2^{*}$ & $1,76 \pm 0,2$ & $1,88 \pm 0,3^{\sharp}$ & $2,02 \pm 0,3^{*}$ \\
Extensores de joelho & $315,7 \pm 48$ & RI1 & RI2 & RI3 & RI1 & RI2 & RI3 \\
\hline
\end{tabular}

* Maior que BB2 e BB1 ( $p<0,001) ;{ }^{*}$ Maior que BB1 $(p<0,001) ;{ }^{\ddagger}$ Maior que RI2 e RI3 $(p<0,001) ;{ }^{+}$Maior que RI3 (p<0,001).
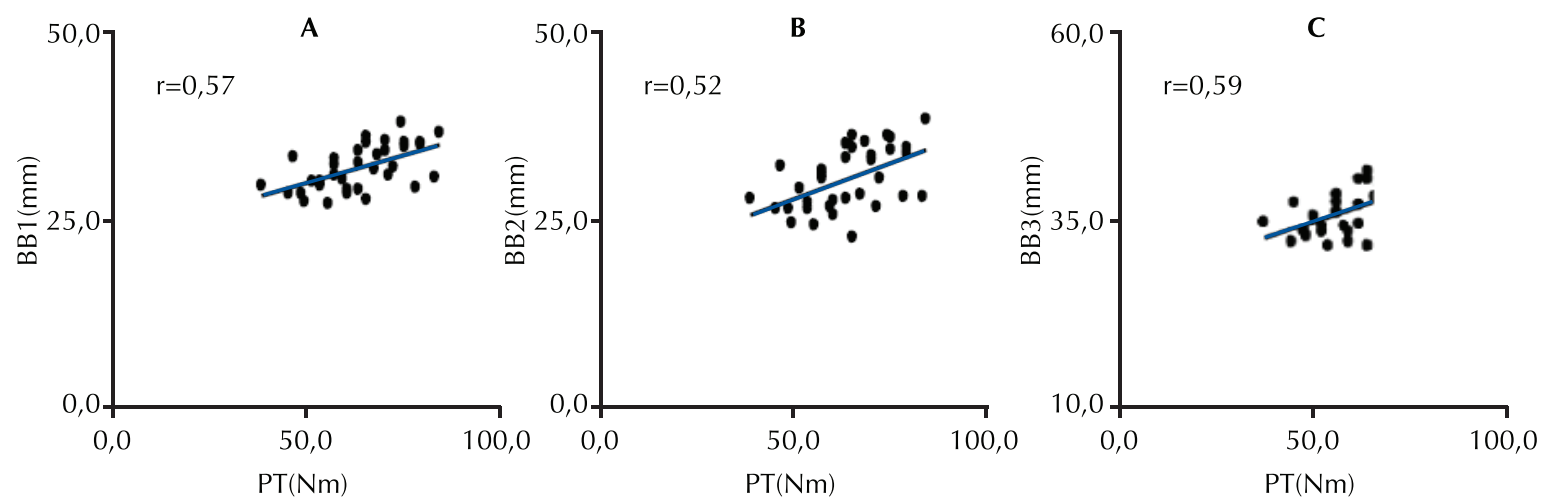

Figura 3. Correlação entre os pontos BB1, BB2, BB3 (mm) e PT (N.m) isométrico de flexão de cotovelo. 

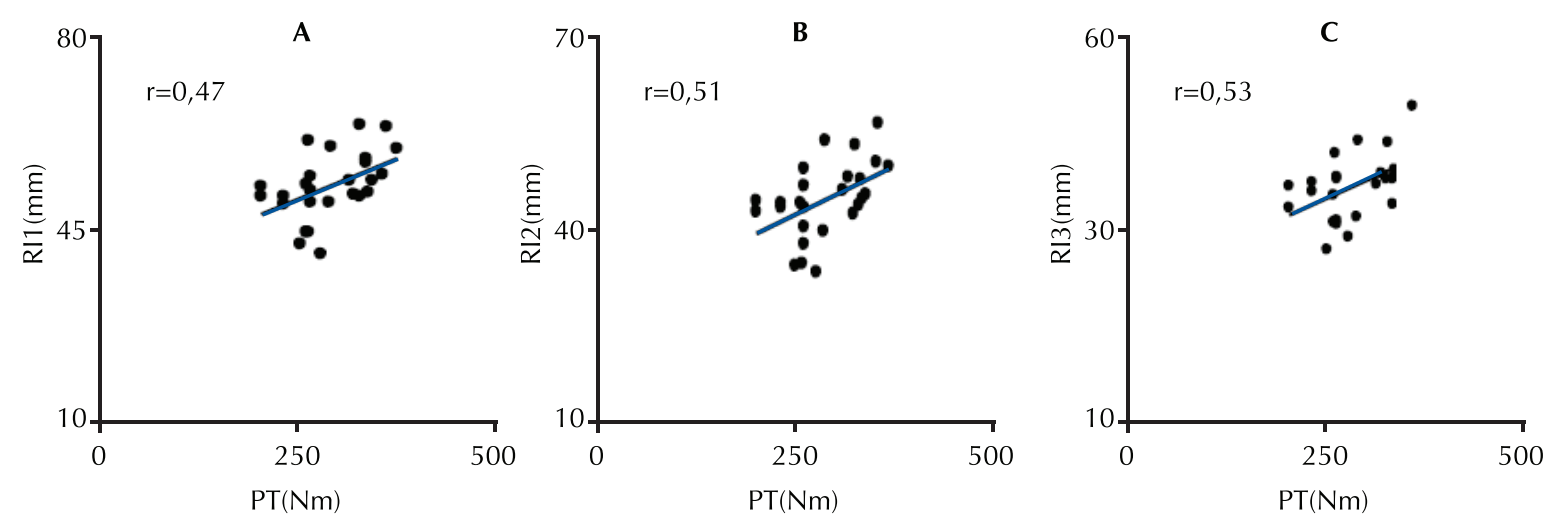

Figura 4. Correlação entre os pontos RI1, RI2, RI3 (mm) e PT (N.m) isométrico de extensão de joelho.

\section{DISCUSSÃO}

O objetivo do presente estudo foi investigar a ocorrência ou não de diferenças entre a EM mensurada em três diferentes pontos nos músculos flexores de cotovelo e extensores de joelho, bem como da QM calculada a partir destas medidas de espessura e dos valores relativos à produção de força isométrica destes músculos. E ainda, pesquisar a correlação das medidas da EM com os valores da produção de força isométrica. Os resultados mostraram que há diferença significativa $(p<0,001)$ entre os valores da EM e da QM obtida a partir de diferentes pontos de medida, bem como que houve correlação dos valores de todos os pontos de avaliação da EM dos flexores de cotovelo (BB1, BB2, BB3) e dos extensores de joelho (RI1, RI2, RI3) com a produção de força isométrica de flexão de cotovelo $(\mathrm{p}<0,05)$ e extensão de joelho $(\mathrm{p}<0,01)$, respectivamente.

A análise de imagens por meio de ultrassonografia com o objetivo de observar sua relação com a produção de força foi, primeiramente, realizada por Ikai e Fukunaga ${ }^{20,21}$. Posteriormente, vários estudos utilizaram imagens obtidas por ultrassonografia ${ }^{11,18,22}$, ressonância magnética ${ }^{8}$ e tomografia computadorizada ${ }^{23}$ para análise das adaptações morfológicas. Especificamente, tratando-se da mensuração da EM por ultrassonografia, tem-se utilizado diferentes pontos de medida ${ }^{10,17}$. Sendo assim, identificar se há semelhança entre os valores de diferentes pontos de medida da EM, como também se estes valores têm correlação com a produção de força muscular, proporcionaria uma importante contribuição metodológica quando a ultrassonografia for utilizada como método de análise. Da mesma forma, a avaliação da QM pode ser mais adequada, considerando-se estas informações.

Os resultados do presente estudo demonstraram que o ponto de mensuração da EM afeta diretamente os resultados de flexores de cotovelo e extensores de joelho. Para o nosso conhecimento, esse é o primeiro estudo que mensurou e comparou a EM dos flexores de cotovelo em mais de um ponto. Como sugerido por Blazevich et al..24, a análise da EM em mais de um ponto possibilitaria uma verificação mais detalhada das adaptações morfológicas ocorridas ao longo do músculo, sobretudo, em decorrência do treinamento realizado em estudos longitudinais. Nesta mesma perspectiva, Narici et $\mathrm{al}^{25}$, utilizando a ressonância magnética como método de análise, constataram que a hipertrofia não ocorre de maneira similar ao longo do músculo. Tais evidências indicam a necessidade da EM ser avaliada em vários pontos do músculo, sobretudo, em estudos longitudinais. A verificação de que os valores de EM ao longo dos músculos extensores do joelho variam significativamente já havia sido previamente estabelecida ${ }^{4,24,26}$. Esses autores constataram que a EM ao longo do Reto da Coxa varia significativamente, o mesmo não ocorrendo, na mesma proporção, para a EM do Vasto Intermédio. Nesse sentido, a variação dos valores da EM nos três pontos da coxa, observadas neste estudo, parece ter sido mais influenciada pela morfologia do músculo Reto da Coxa do que do músculo Vasto Intermédio.

Os resultados do presente estudo demonstraram existir diferença significativa $(p<0,001)$ entre os valores de QM obtido a partir da EM dos flexores de cotovelo e extensores do joelho. A QM parece ser influenciada pelo envelhecimento ${ }^{12}$, pelo treino de força ${ }^{13}$, e pelo nível de treinamento dos sujeitos ${ }^{7}$, e vem sendo apresentada como uma variável que se associa melhor com a capacidade funcional do que com a força muscular isolada ${ }^{12,13}$. Assim, diferentes pontos em que a EM é mensurada apresentam diferentes valores de QM, fato que ressalta a importância do controle do local de avaliação da EM. Dessa maneira, o controle do local de avaliação da 
EM por meio de ultrassonografia é extremamente importante em estudos longitudinais que pretendem investigar adaptações na EM e na QM, uma vez que a alteração desses pontos, tanto no Bíceps Braquial e Braquial quanto no Reto da Coxa e Vasto Intermédio, pode alterar os resultados encontrados, mesmo que nenhuma alteração real tenha ocorrido. No entanto, como mencionado acima, avaliar a EM, em diferentes pontos, é necessário para uma análise mais detalhada das adaptações morfológicas em resposta ao treinamento ${ }^{24}$. Nesta perspectiva, a utilização de mapas de avaliação, como por exemplo, os propostos por Narici et $\mathrm{al}^{29}$, podem ser uma alternativa para o controle dos pontos de avaliação da EM em estudos longitudinais, podendo reduzir os erros na reavaliação dos locais (pontos) de avaliação.

Como mostrado nos resultados, os pontos de avaliação da EM utilizados demonstraram correlação significativa e semelhante com a produção de força isométrica. Outros autores já haviam evidenciado correlação significativa entre EM e produção de força, como Fukunaga et al.? que observaram correlação de $r=0,78$ entre o volume muscular, estimado por meio da EM, dos flexores de cotovelo com a produção de força isométrica em sujeitos jovens não atletas. Além disso, os pontos utilizados para avaliação da EM dos extensores de joelho (RI1, RI2 e RI3), do presente estudo, mostraram correlação significativa com o valor de produção de força isométrica. Provavelmente, em virtude da EM utilizada no presente estudo ter incluído a espessura dos músculos Reto da Coxa e principalmente, a do Vasto Intermédio, uma vez que já foi demonstrado que durante uma contração isométrica de extensão de joelho o Vasto Intermédio é o músculo que apresenta a maior ativação e produção de força isométrica ${ }^{28}$. Esses resultados mostraram que, mesmo havendo diferença significativa entre os valores de EM dos diferentes pontos dos flexores de cotovelo e entre os diferentes pontos dos extensores de joelho, todos apresentaram correlação com os valores de produção de força isométrica. Assim, todos os pontos utilizados no presente estudo parecem ser seguros para a mensuração da EM dos músculos relacionados, uma vez que essas espessuras podem explicar, ao menos em parte, a capacidade de produção de força muscular.

Assim, os resultados do presente estudo demonstram que o local do ponto de mensuração da EM é importante quando se pretende observar adaptações morfológicas, sobretudo, pelo fato de que não somente a EM é influenciada pelo local da avaliação em que é mensurada, mas também a QM. Entretanto, os valores da EM obtidos nos diferentes pontos de medida não afetam a sua correlação com a produção de força isométrica.

\section{CONCLUSÃO}

A determinação adequada e criteriosa dos locais de avaliação da EM é uma variável metodológica importante de ser considerada, quando se objetiva quantificar as adaptações morfológicas em resposta ao treino com a utilização do método ultrassonografia, visto que variações nos pontos em que esta é mensurada podem comprometer ou modificar os resultados obtidos. Assim, a utilização de referências anatômicas adequadamente definidas e apropriadas como, por exemplo, de mapas de avaliação, podem auxiliar no controle do local de avaliação da EM em estudos longitudinais e experimentais. Além disso, independentemente do ponto de mensuração utilizados no presente estudo, os valores da EM dos músculos flexores de cotovelo e extensores de joelho associam-se aos valores de produção de força isométrica destes músculos.

\section{REFERÊNCIAS BIBLIOGRÁFICAS}

1. Sale DG, Martin JE, Moroz DE. Hypertrophy without increased isometric strength after weight training. Eur J Appl Physiol 1992;64(1):51-4.

2. Thortensson A, Grimby G, Karlsson J. Force-velocity relations and fiber composition in human knee extensor muscles. J Appl Physiol 1976;40(1):12-6.

3. Jones EJ, Bishop PA, Woods AK, Green JM. Cross-sectional area and muscular strength. Sports med 2007;38(12):987-94.

4. Blazevich AJ, Gill ND, Zhou S. Intra- and intermuscular variation in human quadriceps femoris architecture assessed in vivo. J Anat 2009;209(3):289-310.

5. Abe T, Dehoyos DV, Pollock ML, Garzaralle L. Time course for strength and muscle thickness changes following upper and lower body resistance training in men and women. anatomy 2000;81(3):174-80.

6. Alegre LM, Jiménez F, Gonzalo-orden JM, Martín-acero $\mathrm{R}$, Agudo X. Effects of dynamic resistance training on fascicle length and isometric strength. J sports sci 2006; 24(5):501-508.

7. Fukunaga T, Miyatani M, Tachi M, Kawakami Y, Kanehisa H., Muscle volume is a major determinant of joint torque in humans. Acta physiol scand 2001;172 (4):249-55.

8. Aagaard P, Andersen JL, Dyhre-Poulsen P, Leffers AM, Wagner A, Magnusson PS, et al. A mechanism for increased contractile strength of human pennate muscle in response to strength training: changes in muscle architecture. J Physiol 2001;534 (2): 613-23. 
9. Reeves ND, Narici MV. Behavior of human muscle fascicles during shortening and lengthening contractions in vivo. Eur J Appl Physiol 2003; 95(3):1090-6.

10. Starkey DB, Pollock ML, Ishida Y, Welsch MA, Brechue WF, Graves JE, er al., Effect of resistance training volume on strength training and muscle thickness. Med Sci Sports Exerc 1996;28(10):1311-19.

11. Nogueira W, Gentil P, Mello SNM, Bezzera AJC, Bottaro M., Effects of Power training on muscle thickness of older men. Int J Sports Med 2009;30(3):200-4.

12. Lynch NA, Metter EJ, Lindle RS, Fozard JL, Tobin TA, ROT JL, et al. Muscle quality. I. Age-associated differences between arm and leg muscle groups. J Appl Physiol 1999;86(1):188-94.

13. Tracy BL, Ivey FM, Martel GF, Lemmer JT, Siegel EL, Metter EJ, et al. Muscle quality. II. Effects of strength training in 65- to 75-yr-old men and women. J Appl Physiol 1999;86(1):195-201.

14. Erskine RM, Jones DA, Maganaris CN, Degens H. In vivo specific tension of the human quadriceps femoris muscle. Eur J Appl Physiol 2009;106(6):827-38.

15. Abe T, Kawakami Y, Gunji A, Fukunaga T. Effects of 20 days bed rest on muscle morphology. Journal of gravitational physiology: J Int Society Gravit Physiol 1997;4(1):10-4.

16. Miyatani M, Kanehisa H, Kawakami MIY, Fukunaga $\mathrm{T}$. The accuracy of volume estimates using ultrasound muscle thickness measurements in different muscle groups. Eur J Appl Physiol 2004;91(2-3):264-72.

17. Mikkola J, Rusko H, Nummela A, Pollari T, Häkkinen $\mathrm{K}$. Concurrent endurance and explosive type strength training improves neuromuscular and anaerobic characteristics in young distance runners. Int J Sports Sci 2006;28(7):602-11.

18. Simão R, Belmiro S, De Salles F, Oliveira LF, MattaT, Miranda F, Miranda H, Costa PB. Influence of exercise order on maximum strength and muscle thickness in untrained men. J Sports Sci Med 2010;9 (1):1-7.

19. Chilibeck PD, Stride D, Farthing JP, Burke DG., Effect of creatine ingestion after exercise on muscle thickness in males and females. Med Sci Sports Exerc 2004; 36(10):1781-8.

20. Ikai M, Fukunaga T. Calculation of muscle strength per unit cross-sectional area of human muscle by muscle by means of ultrasonic measurement Eur J Appl Physiol Occup Physiol 1968;26(1):26-32.

21. Ikai M, Fukunaga T. A study on training effect on strength per unit cross-sectional area of muscle by means of ultrasonic measurement. Eur J Appl Physiol Occup Physiol 1970;28(3):173-80.
22. Häkkinen K, Kallinen M, Izquiero M, Jokelainen K, Lassila $\mathrm{H}$, Mälkiä E, et al. Changes in agonist-antagonist EMG, muscle CSA,and force during strength training in middle-aged and older people. J Appl Physiol 1998;84(4):1341-9.

23. Ferri A, Narici M, Grassi B, Pousson M. Neuromuscular recovery after a strength training session in elderly people. Eur J Appl Physiol 2006;97(3):272-9.

24. Blazevich AJ, Gill ND, Bronks R, Newton RU., Training-specific muscle architecture adaptation after 5-wk training in athletes. Med Sci Sports Exerc 2003;35(12):2013-22

25. Narici MV, Landoni L, Minetti AE. Assessment of human knee extensor muscles stress from in vivo physiological cross-sectional area and strength measurements. Eur J Appl Physiol Occup Physiol 1992;65(2):438-44.

26. Scott SH, Enstrom CE, Loeb GE. Morphometry of human thigh muscles. Determination of fascicle architecture by magnetic resonance imaging. J Anat 1993; 182(2):249-57.

27. Stumbo TA, Merriam S, Nies K, Smith A, Spurgeon D, Weir JP. The effect of hand-grip stabilization on isokinetic torque at the knee. J Strength Cond Resh 2001;15(3):372-7.

28. Zhang $1 Q$, Wange GW, Press JM, Kohn JL. In vivo load sharing among the quadriceps components. J Orthop Res 2003;21(3):565-71.

29. Narici MV, Hoppeler H, Kayser B, Landoni L, Claassen $\mathrm{H}$, Gavardi C, et al. Human quadriceps cross-sectional area, torque and neural activation during 6 months strength training. Acta Physiol Scand 1996;157(2):175-86.

\section{Endereço para correspondência}

Ronei Silveira Pinto

Escola de Educação Física da UFRGS.

Laboratório de Pesquisa do Exercício (LAPEX).

Rua Felizardo, 750, CEP: 90690-200 - Porto Alegre, RS. Brasil.

E-mails: regis.radaelli@hotmail.com; ronei.pinto@ufrgs.br 